\title{
Identificación temprana de niños y niñas sobresalientes en preescolar
}

\section{Pedro Covarrubias Pizarro}

Profesor investigador Benemérita y Centenaria Escuela Normal del Estado de Chihuahua

\section{Resumen}

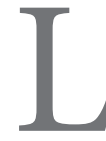

a identificación temprana de niñas y niños sobresalientes en preescolar, tuvo como marco de acción El Proyecto 2009: Atención a niños, niñas y jóvenes con aptitudes sobresalientes, durante el ciclo escolar 20082009, y fue el resultado de una política Nacional, que pretendía impulsar el desarrollo de esta población, por medio de estrategias pertinentes de identificación e intervención. Dentro de un marco de Integración Educativa, la Subsecretaría de Educación Pública, a través del Programa de Fortalecimiento de la Educación Especial y de la Integración Educativa, emitió lineamientos y programas específicos para dar respuesta a las necesidades educativas especiales asociadas a las aptitudes sobresalientes.

En enero de 2009, se inició en el Estado de Chihuahua la planeación de estrategias que permitieran impulsar los grandes retos de la atención a los sobresalientes, y sobre todo, iniciar con la detección temprana en preescolar, nivel educativo en donde no se contaba con antecedentes de intervención para este sector de la población. Bajo este escenario se elaboró el proyecto Identificación Temprana de niñas y niños sobresalientes en Preescolar, en colaboración con el Centro Huerta del Rey ${ }^{1}$, de Valladolid España, coordinado por la Dra. Yolanda Benito y la Secretaría de Educación y Cultura, a través del Programa Estatal de Fortalecimiento a la Educación Especial y la Integración Educativa.

\section{Introducción}

Históricamente, México inicia con la atención de las y los alumnos sobresalientes, en la década de los 80 , sin embargo, no es hasta el año 2007, cuando se emite una Propuesta Nacional para todas las escuelas de educación básica (en el nivel de primaria), que cuentan 
con la asesoría de las Unidades de Apoyo a la Educación Regular (UsAer). Dentro de este margen de acción, se plantearon metas para la ampliación de la cobertura y sobre todo, para iniciar los trabajos en los niveles de preescolar y secundaria.

Durante el ciclo escolar 2006 - 2007, la Subsecretaría de Educación Básica, presentó los siguientes datos nacionales relacionados con la atención de las y los alumnos con aptitudes sobresalientes:

D Existen 20,785 escuelas de educación básica con apoyo de Usaer.

De atienden 166,612 alumnos y alumnas en educación especial.

- Del total de escuelas, solo 220 reportaban atención a niños y niñas sobresalientes.

- Del total de alumnas y alumnos atendidos por medio de Usaer, solo 1921 eran con aptitudes sobresalientes. (Fuente: Programa Estatal para el Fortalecimiento de la Integración Educativa de la Educación Especial y de la Integración educativa).

Esta situación puso en vulnerabilidad al grupo de estudiantes de Educación Básica que presentan alguna aptitud sobresaliente, ya que solo se estaban atendiendo a menos del 1\% de esta población. Bajo este escenario, el Programa Sectorial de Educación 2008 - 2012 retoma como uno de sus objetivos centrales el impulso a la atención de las y los niños con aptitudes sobresalientes, como una estrategia fundamental para elevar los indicadores educativos y en apego a la atención a la diversidad.

Este marco normativo nacional y las políticas educativas estatales, permitieron plantear el Proyecto Identificación Temprana de niñas y niños sobresalientes en Preescolar, en colaboración con Centro Huerta del Rey.

El trabajo propuesto para iniciar con las actividades de identificación temprana en preescolar, representó la colaboración de diferentes autoridades estatales pertenecientes a los dos subsistemas del Estado de Chihuahua, encabezados por la Lic. María Guadalupe Chacón Monárrez, Secretaria de Educación y Cultura en ese momento y la Maestra Irma Otilia Ayala Domínguez quien fungía como Coordinadora del Programa Estatal de Fortalecimiento de la Educación Especial y de la Integración Educativa. Se contó con el aval de todos los funcionarios intermedios relacionados con el nivel educativo, tanto de Educación Básica como de Educación Especial de ambos subsistemas.

El propósito de la Identificación Temprana de niñas y niños sobresalientes en Preescolar, tuvo sustento en las políticas estatales y nacionales. Dentro del Programa Estatal de Fortalecimiento de la Educación Especial y de la Integración Educativa 2005 - 2010, se delimitaban siete líneas de acción:

- Línea 1. Lineamientos generales de atención y promoción educativa de los alumnos con aptitudes sobresalientes y/o talentos específicos.

- Línea 2. Propuesta de atención educativa para alumnos con aptitudes sobresalientes y/o talentos específicos.

- Línea 3. Actualización y capacitación del personal docente de educación regular y especial, en los temas de Aptitudes Sobresalientes (AS) y/o Talentos Específi$\cos$ (TE).

- Línea 4. Información y sensibilización a la 
comunidad acerca de temas relacionados con las AS y/o TE.

- Línea 5. Dotación de recursos y materiales específicos para promover el desarrollo de actividades relacionados con la ciencia, el deporte y las artes en las escuelas de educación básica.

- Línea 6. Participación activa de las familias en el desarrollo de las potencialidades de sus hijos.

- Línea 7. Vinculación intra e interinstitucional entre los distintos sectores y organizaciones de la sociedad civil para apoyar el proceso de atención de los alumnos con AS y/o TE.

Uno de los principales retos en la atención a niños y niñas con aptitudes sobresalientes y/o talentos específicos en el Estado de Chihuahua era ampliar la cobertura y fortalecer el proceso de atención en preescolar. De la línea 2 , se derivaron varias estrategias que dieron forma $y$ vida al proyecto de investigación con el Centro Huerta del Rey.

\section{Fundamentos teóricos}

Como parte de los elementos teóricos que sustentaron el trabajo, se retomaron algunos de los principios propuestos por la Dra. Yolanda Benito (2009).

Se considera a los alumnos superdotados, considerando el criterio psicométrico de CI igual o superior a 130.

El término superdotación parece adecuado para identificar a las altas habilidades naturales, parcialmente innatas, y que se desarrollan de forma natural mediante procesos madurativos, así como por el uso diario y/o la práctica formal (Gagné, citado por Benito, 2009).
El criterio para determinar la existencia de una sobredotación intelectual es la obtención de una puntuación en rendimiento de la inteligencia conceptual de dos o más desviaciones típicas por encima de la media.

Los tres criterios que parecen ser indicativos de la sobredotación intelectual:

1. La sobredotación intelectual se caracteriza por un funcionamiento intelectual significativo superior a la media.

2. La capacidad intelectual se define como CI, que va asociado a una mayor madurez en los procesos de información, alta motivación para el aprendizaje, creatividad, precocidad y talento.

3. La superdotación intelectual debe manifestarse durante la etapa de desarrollo, desde la concepción hasta los 18 años.

Se piensa que en el niño con sobredotación intelectual, con un CI superior a 130, tiene que observarse un desarrollo y un aprendizaje distinto al del niño normal, siendo más precoz que éste en el desarrollo motor, social, de lenguaje y del aprendizaje.

Las variables que se consideran como posibles indicativos de alta capacidad son:

- Desarrollo motor

- Desarrollo de lenguaje

- Desarrollo cognitivo

- Autoayuda

- Socialización

En México, el concepto de superdotación se traduce como aptitudes sobresalientes, y la definición que estipula la política nacional es: "Los niños, niñas y jóvenes con aptitudes sobresalientes son aquellos capaces de destacar 
significativamente del grupo social y educativo al que pertenecen en uno o más de los campos del quehacer humano como son el científicotecnológico, el humanístico-social, el artístico y el de acción-motriz. Sus características los llevan a mostrar una serie de necesidades específicas que requieren ser atendidas mediante un contexto que sea facilitador del desarrollo de sus habilidades, conocimientos e intereses" (SEP, 2006: 59).

\section{Estrategia metodológica}

La metodología propuesta para llevar a cabo el Proyecto de Identificación Temprana de niñas y niños sobresalientes en Preescolar, se apegó a los criterios estipulados por Centro Huerta del Rey, en donde se marcaron las siguientes acciones:

- Selección de por lo menos 600 alumnos en edad preescolar, para ser valorados por medio de la Tabla de Observación y Desarrollo.

- Aplicación de la Tabla de Observación y Desarrollo a las madres y padres de familia de los alumnos preescolares.

- Valoración del Coeficiente Intelectual por medio de pruebas estandarizadas a las y los alumnos que pasaran el screening, para corroborar la presencia de un CI de 130 puntos o más.

En el estado de Chihuahua se programó la selección de los preescolares por medio de los servicios de educación especial que brindaban atención en los Jardines de Niños, de ambos subsistemas:

Por parte del subsistema estatal se inició el trabajo por medio de seis Unidades de Apoyo a la Educación Regular (UsAer) y la selección de seis preescolares.

Por parte del subsistema federal se inició el trabajo por medio de trece Unidades de Apoyo a Preescolares con Necesidades Educativas Especiales (UAPNEE) y la selección de dieciocho Preescolares

Todos los Jardines de Niños seleccionados, pertenecían al Municipio de Chihuahua, capital del estado.

Como parte de las estrategias metodológicas para llevar a cabo el proyecto, se propusieron tres fases de trabajo:

A. Fase de Sensibilización. Esta fase tuvo como propósito general, bajar la información pertinente a los diferentes actores involucrados en el proceso; se consideraron desde las jefaturas académicas y administrativas, hasta el personal de los preescolares donde se implementaría el trabajo. Su ejecución fue del 10 al 27 de febrero del 2009.

- Reunión con Autoridades de Educación Básica y Elemental en ambos subsistemas

- Reunión con Autoridades de Educación Especial y Educación Preescolar de ambos subsistemas

- Reunión con directoras, inspectoras, supervisoras y jefas de sector de JN seleccionados / directoras de USAER-UAPNEE / Inspectoras-supervisoras de EE

- Reunión con educadoras de los Jardines de Niños seleccionados / Directoras JN y Usaer / UAPNEE de ambos subsistemas

B. Fase de Capacitación. La segunda fase del proyecto consistió en la capacitación para 
el personal de educación preescolar y los servicios de educación especial participantes en el proyecto. En esta fase se contó con la presencia de la Dra. Yolanda Benito del 9 al 13 de marzo del 2009.

Los temas que se abordaron en la semana de capacitación fueron:

1. Diferenciación del concepto sobredotado, genio, prodigio, talento.
2. Conceptualización de la sobredotación intelectual.

3. Método de cribaje o screening / tabla de Observación y Desarrollo.

4. Trastornos asociados con la sobredotación.

5. Diagnóstico diferencial de los alumnos con sobredotación intelectual.

\section{Participantes en la capacitación del subsistema estatal}

Cuadro 1

\begin{tabular}{|c|c|c|c|c|}
\hline Servicio de educación especial & Director/a & Psicólogo/a & Maestra de apoyo & OTROS \\
\hline USAER 7614 & 1 & 1 & 1 & \\
\hline USAER 7064 & 1 & 1 & 1 & \\
\hline USAER 7616 & 1 & 1 & 1 & \\
\hline USAER 7503 & 1 & 1 & 1 & \\
\hline USAER 7601 & 1 & 1 & 1 & 4 \\
\hline USAER 7500 & 1 & 1 & & 2 \\
\hline UTEE/ATP & & & & 1 \\
\hline Coordinadora UTEE & & & & 2 \\
\hline Inspectoras & & & & \\
\hline Coordinador AS Preescolar & & & & \\
\hline TOTAL & 6 & 6 & & \\
\hline
\end{tabular}

\section{Participantes en la capacitación del subsistema federal}

Cuadro 2

\begin{tabular}{|c|c|c|c|c|}
\hline Servicio de educación especial & Director/a & Psicólogo/a & Maestra de apoyo & OTROS \\
\hline UAPNEE 1 & 1 & 1 & 1 & \\
\hline UAPNEE 2 & 1 & 1 & 1 & \\
\hline UAPNEE 3 & 1 & 1 & 1 & \\
\hline UAPNEE 4 & 1 & 1 & 1 & \\
\hline UAPNEE 5 & 1 & 1 & 1 & \\
\hline UAPNEE 6 & 1 & 1 & 1 & \\
\hline UAPNEE 7 & 1 & 1 & 1 & \\
\hline UAPNEE 8 & 1 & 1 & 1 & \\
\hline UAPNEE 9 & 1 & 1 & 1 & \\
\hline UAPNEE 10 & 1 & 1 & 1 & \\
\hline UAPNEE 20 & 1 & 1 & 1 & \\
\hline UAPNEE 21 & 1 & 1 & 1 & \\
\hline UAPNEE 24 & 1 & 1 & 1 & \\
\hline Supervisoras & & & & 4 \\
\hline Asesores Técnicos & & & & 4 \\
\hline Coordinadora AS Preescolar & & & & \\
\hline TOTAL & 13 & 13 & 13 & \\
\hline
\end{tabular}


REVISTA DE INVESTIGACIÓN EDUCATIVA DE LA REDIECH N. 3

Invitados: Personal del Centro de Recursos e Información para el Fortalecimiento de la Integración Educativa Especial de Cd. Juárez, Chihuahua (6 asistentes), asesores del Equipo de Apoyo a la Docencia (2 asistentes) y la Coordinadora y asesores del Programa Estatal para el Fortalecimiento de la Integración Educativa de la Educación Especial (2 asistentes).

En total asistieron a la capacitación 84 personas (ver cuadros 1 y 2).

Fase Operativa. La tercera y última fase tuvo como propósito general, llevar a cabo el trabajo propuesto para la identificación temprana de las y los alumnos sobresalientes en preescolar.

\begin{tabular}{|c|c|c|c|}
\hline & & & Cuadro 3 \\
\hline \multicolumn{4}{|c|}{ APLICACIÓN DE LA TABLA DE OBSERVACIÓN Y DESARROLLO } \\
\hline $\begin{array}{l}\text { Informar a padres y madres } \\
\text { de familia }\end{array}$ & $\begin{array}{l}\text { Aplicación } \\
\text { en grupos } \\
\text { de } 1^{\circ} \text { y } 2^{\circ} \\
\text { grado de } \\
\text { preescolar }\end{array}$ & $\begin{array}{l}\text { Aplicación por } \\
\text { medio de las } \\
\text { maestras de apoyo } \\
\text { y psicólogas }\end{array}$ & $\begin{array}{l}\text { Fecha: } 17 \text { / } 23 \\
\text { de marzo } 2009\end{array}$ \\
\hline \multicolumn{4}{|c|}{ SCREENING } \\
\hline $\begin{array}{l}\text { Revisión de las Tablas de } \\
\text { Observación y Desarrollo }\end{array}$ & \multicolumn{2}{|c|}{$\begin{array}{l}\text { Preselección de las y los alumnos } \\
\text { por medio del método de cribaje } \\
\text { Preselección de las y los alumnos } \\
\text { por medio de la nominación por } \\
\text { criterio }\end{array}$} & $\begin{array}{l}\text { Fecha: } 24 \text { / } 31 \\
\text { de marzo } 2009\end{array}$ \\
\hline \multicolumn{4}{|c|}{ APLICACIÓN DE EVALUACIONES } \\
\hline \multicolumn{2}{|c|}{$\begin{array}{l}\text { Aplicación de instrumentos estandarizados } \\
\text { para la valoración del Coeficiente Intelectual }\end{array}$} & $\begin{array}{l}\text { Integración de los } \\
\text { resultados }\end{array}$ & $\begin{array}{l}\text { Fecha: del } 1^{\circ} \text { al } \\
19 \text { de junio }\end{array}$ \\
\hline \multicolumn{2}{|c|}{ CONCENTRADO DE LA INFORMACIÓN } & \multicolumn{2}{|c|}{ ENVÍO DE RESULTADOS A ESPAÑA } \\
\hline \multicolumn{2}{|l|}{ Fecha: 22 / 26 de Junio 2009} & \multicolumn{2}{|c|}{ Fecha: 29 de junio / 2 julio 2009} \\
\hline
\end{tabular}

\section{Sistematización de los datos}

En el presente apartado se integran los resultados obtenidos durante en el Proyecto Identificación temprana de niñas y niños sobresalientes en Preescolar. En un primer mo- mento se incluyen los cuadros que concentran la información general por subsistema. Posteriormente se presentan las gráficas correspondientes a los resultados y la interpretación de las mismas. 
Cuadro 4. Resultados generales de la entidad

\begin{tabular}{|c|c|c|c|c|c|c|c|c|c|c|c|c|c|c|c|}
\hline \multicolumn{3}{|c|}{ Datos generales } & \multicolumn{2}{|c|}{ Sexo } & \multicolumn{3}{|c|}{ Preseleccionados } & \multicolumn{2}{|c|}{ Instrumentos aplicados } & \multicolumn{4}{|c|}{ Resultados obtendios en $\mathrm{Cl}$} & \multirow[b]{2}{*}{ Sin resultados } & \multirow[b]{3}{*}{ Estatal } \\
\hline Preescolar & $\begin{array}{r}\text { Servicio de } \\
\text { E. Especial }\end{array}$ & $\begin{array}{c}\text { Niños y niñas } \\
\text { valorados }\end{array}$ & H & M & $\begin{array}{l}\text { Por } \\
\text { cribaje }\end{array}$ & $\begin{array}{l}\text { Nominación } \\
\text { por criterio }\end{array}$ & Total & $\begin{array}{c}\text { WPPSI } \\
\text { aplicados }\end{array}$ & $\begin{array}{l}\text { TERMAN } \\
\text { aplicados }\end{array}$ & $\begin{array}{c}\text { Rango } 90 \\
109\end{array}$ & $\begin{array}{c}\text { Rango } 110 \\
-119\end{array}$ & $\begin{array}{c}\text { Rango } 120 \\
-129\end{array}$ & $\begin{array}{l}\text { Cl } 1300 \\
\text { más }\end{array}$ & & \\
\hline Godofredo de Koster 1356 & 7614 & 61 & 33 & 28 & 8 & 0 & 8 & 6 & 2 & 3 & 3 & 2 & 0 & 0 & \\
\hline Octavio Paz 1401 & 7064 & 81 & 44 & 37 & 3 & 1 & 4 & 3 & 0 & 2 & 0 & 1 & 0 & 1 & \\
\hline Carlos Montemayor 1350 & 7616 & 50 & 30 & 20 & 8 & 8 & 16 & 16 & 0 & 13 & 2 & 1 & 0 & 0 & \\
\hline 24 de Febrero 1389 & 7503 & 46 & 20 & 26 & 0 & 6 & 6 & 6 & 0 & 0 & 1 & 2 & 3 & 0 & \\
\hline Quijote de la Mancha 8106 & 7601 & 37 & 19 & 18 & 0 & 0 & 0 & 0 & 0 & 0 & 0 & 0 & 0 & 0 & \\
\hline Ana Mará Gallaga 1003 & 7500 & 58 & 34 & 24 & 17 & 1 & 18 & 15 & 0 & 8 & 1 & 5 & 1 & 3 & \\
\hline $\begin{array}{l}\text { María Elena Chánez } \\
\text { 08DJN0018-Y }\end{array}$ & $\begin{array}{c}\text { UAPNEE } \\
1\end{array}$ & 110 & 56 & 54 & 3 & 0 & 3 & 2 & 0 & 1 & 0 & 1 & 0 & 1 & Federal \\
\hline $\begin{array}{l}\text { Juan Alanis } \\
\text { 08DJN0013C }\end{array}$ & $\mid \begin{array}{c}\text { UAPNEE } \\
2\end{array}$ & 28 & 17 & 11 & 1 & 0 & 1 & 1 & 0 & 0 & 0 & 0 & 1 & 0 & \\
\hline $\begin{array}{l}\text { Margarita Hermosillo de } \\
\text { Campos 08DJN0055-B }\end{array}$ & $\begin{array}{c}\text { UAPNEE } \\
3\end{array}$ & 45 & 23 & 22 & 3 & 1 & 4 & 4 & 0 & 1 & 2 & 0 & 0 & 1 & \\
\hline $\begin{array}{l}\text { Luz María Serradel } \\
\text { 08DJN0117-Y }\end{array}$ & UAPNEE & 1 & 0 & 1 & 1 & 0 & 1 & 1 & 0 & 0 & 0 & 0 & 1 & 0 & \\
\hline $\begin{array}{l}\text { Ramona Cortés Rodriguez } \\
\text { 08DJN0879-D }\end{array}$ & 4 & 78 & 37 & 41 & 2 & 2 & 4 & 4 & 0 & 0 & 1 & 3 & 0 & 0 & \\
\hline $\begin{array}{l}\text { Teporaca } \\
\text { 08DJN2001-L }\end{array}$ & UAPNEE & 1 & 1 & 0 & 1 & 0 & 1 & 1 & 0 & 0 & 0 & 1 & 0 & 0 & \\
\hline $\begin{array}{l}\text { María Edmee Álvarez } \\
\text { 08DJN2115-N }\end{array}$ & 5 & 62 & 39 & 23 & 1 & 1 & 2 & 2 & 0 & 0 & 2 & 0 & 0 & 0 & \\
\hline $\begin{array}{l}\text { Rayenari } \\
\text { 08DJN0733-J }\end{array}$ & $\mid \begin{array}{c}\text { UAPNEE } \\
6\end{array}$ & 36 & 13 & 23 & 4 & 0 & 4 & 4 & 0 & 0 & 2 & 1 & 0 & 1 & \\
\hline $\begin{array}{l}\text { Carmen Calderón Córdova } \\
\text { 08DJN0062-K }\end{array}$ & $\begin{array}{c}\text { UAPNEE } \\
8\end{array}$ & 42 & 18 & 24 & 0 & 0 & 0 & 0 & 0 & 0 & 0 & 0 & 0 & 0 & \\
\hline $\begin{array}{l}\text { Casa Cuna DIF } \\
\text { 08DJN2147-F }\end{array}$ & & 19 & 8 & 11 & 4 & 0 & 4 & 4 & 0 & 3 & 0 & 0 & 0 & 1 & \\
\hline $\begin{array}{l}\text { Juan Amos Comenio } \\
\text { 08DJN0210-D }\end{array}$ & $\begin{array}{c}\text { UAPNEE } \\
9\end{array}$ & 2 & 2 & 0 & 1 & 0 & 1 & 1 & 0 & 0 & 0 & 1 & 0 & 0 & \\
\hline Gabriela Mistral 08DJN3655-F & & 1 & 1 & 0 & 0 & 1 & 1 & 1 & 0 & 0 & 0 & 0 & 1 & 0 & \\
\hline $\begin{array}{l}\text { María Boschetti Alberti } \\
\text { 08DJN2000-M }\end{array}$ & $\begin{array}{c}\text { UAPNEE } \\
10\end{array}$ & 43 & 24 & 19 & 0 & 1 & 1 & 1 & 0 & 0 & 0 & 0 & 1 & 0 & \\
\hline $\begin{array}{l}\text { Teresa de Calcuta 08DJN2004- } \\
\text { I }\end{array}$ & $\mid \begin{array}{c}\text { UAPNEE } \\
20\end{array}$ & 23 & 11 & 12 & 1 & 1 & 2 & 2 & 0 & 0 & 2 & 0 & 0 & 0 & \\
\hline $\begin{array}{l}\text { Elisa Griensen } \\
\text { 08DJN2113-P }\end{array}$ & \begin{tabular}{|c|} 
UNIDAD \\
21
\end{tabular} & 48 & 26 & 22 & 2 & 0 & 2 & 2 & 0 & 1 & 0 & 0 & 1 & 0 & \\
\hline $\begin{array}{l}\text { Niños Héroes de Chapultepec } \\
\text { 08DJN0048-S }\end{array}$ & $\begin{array}{c}\text { UNIDAD } \\
24\end{array}$ & 23 & 9 & 14 & 4 & 3 & 7 & 7 & 0 & 4 & 2 & 0 & 1 & 0 & \\
\hline Colegio Particualr & $\begin{array}{l}\text { UAPNEE } \\
\text { Coord. }\end{array}$ & 1 & 0 & 1 & 0 & 1 & 1 & 1 & 0 & 0 & 0 & 1 & 0 & 0 & \\
\hline TOTALES & & 940 & 492 & 448 & 65 & 27 & 92 & 85 & 2 & 36 & 19 & 19 & 10 & 8 & 92 \\
\hline
\end{tabular}


REVISTA DE INVESTIGACIÓN EDUCATIVA DE LA REDIECH N. 3

Cuadro 5. Base de datos del subsistema estatal

\begin{tabular}{|c|c|c|c|c|c|c|c|c|c|c|c|c|c|c|}
\hline \multicolumn{3}{|c|}{ Datos Generales } & \multicolumn{2}{|c|}{ Sexo } & \multirow[b]{2}{*}{ Grado } & \multicolumn{3}{|c|}{ Fecha de nacimiento } & & \multicolumn{4}{|c|}{ Resultados Screening } & \\
\hline Serv. & No & Folio & $\mathbf{M}$ & $\mathbf{H}$ & & dd & $\mathbf{m m}$ & $\mathbf{a a}$ & Edad & NO & SI & Nom & $\mathbf{C I}$ & OBSERV. \\
\hline \multirow{8}{*}{$\begin{array}{l}7 \\
6 \\
1 \\
4\end{array}$} & 1 & 0007 & 1 & & 10 & 20 & Nov & 2005 & 3.07 & & $\mathrm{X}$ & & 109 & TERMAN \\
\hline & 2 & 0008 & 1 & & 10 & 06 & Feb & 2005 & 4.04 & & $\mathrm{x}$ & & 119 & \\
\hline & 3 & 0009 & & 1 & 10 & 24 & Oct & 2005 & 3.08 & & $x$ & & 120 & TERMAN \\
\hline & 4 & 0023 & & 1 & 20 & 09 & Ago & 2004 & 4.10 & & $\mathrm{X}$ & & 127 & \\
\hline & 5 & 0046 & 1 & & 20 & 17 & Feb & 2004 & 5.04 & & $\mathrm{x}$ & & 92 & \\
\hline & 6 & 0047 & 1 & & 20 & 14 & Ene & 2004 & 5.05 & & $\mathrm{x}$ & & 119 & \\
\hline & 7 & 0049 & 1 & & 20 & 20 & Feb & 2004 & 5.04 & & $\mathrm{x}$ & & 99 & \\
\hline & 8 & 0059 & & 1 & 30 & 23 & Jun & 2003 & 6.00 & & $x$ & & 116 & \\
\hline \multirow{4}{*}{$\begin{array}{l}7 \\
0 \\
6 \\
4 \\
\end{array}$} & $\overline{9}$ & 0076 & 1 & & 20 & 14 & $\overline{A b r}$ & 2004 & 5.02 & & $\bar{X}$ & & 101 & \\
\hline & 10 & 0079 & 1 & & 30 & 22 & May & 2003 & 6.01 & $x$ & & $\bar{x}$ & 125 & \\
\hline & 11 & 0090 & 1 & & 20 & 25 & Dic & 2004 & 4.06 & & $x$ & & 104 & \\
\hline & 12 & 0133 & & 1 & 10 & 8 & Ene & 2005 & 4.05 & & $x$ & & & No accedió \\
\hline \multirow{16}{*}{$\begin{array}{l}7 \\
6 \\
1 \\
6\end{array}$} & 13 & 0168 & 1 & & 20 & 28 & Nov & 2004 & 4.07 & & $x$ & & 92 & \\
\hline & 14 & 0170 & 1 & & 10 & 03 & $\mathrm{Abr}$ & 2005 & 4.02 & $\mathrm{x}$ & & $\bar{x}$ & 109 & \\
\hline & 15 & 0175 & 1 & & 10 & 04 & Ago & 2005 & 3.10 & & $x$ & & 108 & \\
\hline & 16 & 0178 & & 1 & 10 & 19 & Dic & 2005 & 3.06 & & $\mathrm{X}$ & & 107 & \\
\hline & 17 & 0179 & 1 & & 10 & 30 & $A b r$ & 2005 & 4.02 & & $\mathrm{X}$ & & 85 & \\
\hline & 18 & 0187 & & 1 & 20 & 07 & Mar & 2004 & 5.03 & & $x$ & & 105 & \\
\hline & 19 & 0190 & & 1 & 20 & 13 & Oct & 2004 & 4.08 & & $x$ & & 106 & \\
\hline & 20 & 0192 & & 1 & 10 & 21 & Jun & 2005 & 4.00 & & $\bar{X}$ & & 123 & \\
\hline & 21 & 0193 & 1 & & 20 & 01 & Ago & 2004 & 4.10 & $x$ & & $\mathrm{X}$ & 105 & \\
\hline & 22 & 0198 & & 1 & 10 & 26 & $\mathrm{Abr}$ & 2005 & 4.02 & $x$ & & $\bar{x}$ & 99 & \\
\hline & 23 & 0201 & & 1 & 20 & 29 & Oct & 2004 & 4.08 & $x$ & & $x$ & 109 & \\
\hline & 24 & 0203 & & 1 & 30 & 22 & Dic & 2003 & 5.06 & $x$ & & $\bar{x}$ & 114 & \\
\hline & 25 & 0204 & & 1 & 10 & 30 & Nov & 2005 & 3.07 & $x$ & & $\bar{x}$ & 98 & \\
\hline & 26 & 0210 & & 1 & 30 & 01 & Feb & 2003 & 6.04 & & $x$ & & 91 & \\
\hline & 27 & 0211 & & 1 & 30 & 18 & Ago & 2003 & 5.10 & $\bar{x}$ & & $\bar{x}$ & 116 & \\
\hline & 28 & 0213 & 1 & & 20 & 09 & Sep & 2004 & 4.09 & $\mathrm{X}$ & & $\bar{X}$ & 99 & \\
\hline \multirow{6}{*}{$\begin{array}{l}7 \\
5 \\
0 \\
3\end{array}$} & 29 & 0227 & & 1 & 20 & 17 & Dic & 2004 & 4.06 & $\bar{x}$ & & $\bar{x}$ & 129 & \\
\hline & 30 & 0230 & 1 & & 20 & 13 & Ago & 2004 & 4.10 & $x$ & & $\mathrm{X}$ & 120 & \\
\hline & 31 & 0245 & & 1 & 20 & 4 & Jun & 2004 & 5 & $x$ & & $\bar{X}$ & 144 & \\
\hline & 32 & 0251 & 1 & & 20 & 16 & Jul & 2004 & 4.11 & $\mathrm{X}$ & & $\bar{X}$ & 133 & \\
\hline & 33 & 0261 & 1 & & 20 & 13 & Ago & 2004 & 4.10 & $x$ & & $\mathrm{X}$ & 113 & \\
\hline & 34 & 0270 & & 1 & 30 & 6 & $\mathrm{Abr}$ & 2003 & 6.02 & $x$ & & $\bar{x}$ & 143 & \\
\hline \multirow{18}{*}{$\begin{array}{l}7 \\
5 \\
0 \\
0\end{array}$} & 35 & 0329 & & 1 & 20 & 6 & Oct & 2004 & 4.08 & & $\bar{x}$ & & 124 & \\
\hline & 36 & 0330 & & 1 & 10 & 1 & Sep & 2005 & 3.09 & & $x$ & & 93 & \\
\hline & 37 & 0331 & & 1 & 10 & 1 & Sep & 2005 & 3.09 & & $x$ & & $\mathbf{x}$ & No aceptó \\
\hline & 38 & 0336 & & 1 & 20 & 17 & Oct & 2003 & 5.08 & & $x$ & & 99 & \\
\hline & 39 & 0337 & & 1 & 10 & 23 & Ago & 2005 & 3. 10 & & $\mathrm{X}$ & & $\mathbf{x}$ & No aceptó \\
\hline & 40 & 0343 & 1 & & 20 & 11 & Ene & 2004 & 5.04 & & $\mathrm{X}$ & & 109 & \\
\hline & 41 & 0344 & 1 & & 20 & 19 & Nov & 2004 & 4.07 & & $\mathrm{X}$ & & 122 & \\
\hline & 42 & 0345 & & 1 & 20 & 8 & $\mathrm{Abr}$ & 2004 & 5.02 & $x$ & & $\bar{x}$ & 117 & \\
\hline & 43 & 0355 & 1 & & 20 & 13 & Abr & 2004 & 5.02 & & $x$ & & 129 & \\
\hline & 44 & 0356 & 1 & & 10 & 14 & Jul & 2005 & 3.11 & & $x$ & & 131 & \\
\hline & 45 & 0357 & & 1 & 20 & 2 & Dic & 2004 & 4. 06 & & $x$ & & 122 & \\
\hline & 46 & 0361 & & 1 & 10 & 11 & Nov & 2005 & 3.07 & & $\mathrm{X}$ & & 109 & \\
\hline & 47 & 0362 & 1 & & 20 & 17 & Jul & 2005 & 3.11 & & $x$ & & $\mathbf{x}$ & Se enfermó \\
\hline & 48 & 0368 & & 1 & 20 & 25 & Ago & 2005 & 3. 10 & & $x$ & & 87 & \\
\hline & 49 & 0371 & 1 & & 10 & 9 & Abr & 2005 & 4.02 & & $x$ & & 102 & \\
\hline & 50 & 0377 & & 1 & 20 & 21 & Ene & 2005 & 4.05 & & $\mathrm{X}$ & & 99 & \\
\hline & 51 & 0381 & 1 & & 20 & 9 & May & 2005 & 4. 02 & & $\mathrm{X}$ & & 128 & \\
\hline & 52 & 0386 & 1 & & 20 & 16 & Feb & 2004 & 5.04 & & $X$ & & 109 & \\
\hline & & & 25 & 27 & & & & & & & 36 & 16 & & \\
\hline & & & & $\overline{52}$ & & & & & & & & 52 & & \\
\hline
\end{tabular}


Cuadro 6. Base de datos del subsistema federal

\begin{tabular}{|c|c|c|c|c|c|c|c|c|c|c|c|c|c|c|}
\hline \multicolumn{3}{|c|}{ Datos Generales } & \multicolumn{2}{|c|}{ Sexo } & \multirow[b]{2}{*}{ Grado } & \multicolumn{3}{|c|}{ Fecha de nacimiento } & \multirow[b]{2}{*}{ Edad } & \multicolumn{4}{|c|}{ Resultados Screening } & \multirow[b]{2}{*}{ OBSERV. } \\
\hline Serv. & No & Folio & $M$ & $\mathrm{H}$ & & dd & $\mathrm{mm}$ & aa & & NO & SI & Nom & $\mathrm{Cl}$ & \\
\hline \multirow{3}{*}{ UAPNEE 1} & 1 & 0405 & & 1 & $1^{\circ}$ & 26 & Jul & 2005 & 3.8 & & $\mathrm{X}$ & & * & BAJA \\
\hline & 2 & 0423 & & 1 & $2^{\circ}$ & 10 & Ago & 2004 & $4.09 / 15$ & & $\mathrm{X}$ & & 107 & \\
\hline & 3 & 0530 & & 1 & $1^{\circ}$ & 10 & Feb & 2005 & $4.03 / 10$ & & $\mathrm{X}$ & & 129 & \\
\hline UAPNEE 2 & 4 & 0545 & & 1 & $2^{\circ}$ & 1 & Ago & 2004 & $4.09 / 12$ & & $x$ & & 141 & \\
\hline \multirow{4}{*}{ UAPNEE 3 - } & 5 & 0591 & & 1 & $2^{\circ}$ & 23 & Jun & 2004 & 4.11 & $\mathrm{x}$ & & $\mathrm{X}$ & 113 & \\
\hline & 6 & 0593 & & 1 & $1^{\circ}$ & 9 & Dic & 2005 & $4.04 / 08$ & & $\mathrm{X}$ & & * & INCUANTIFICABLE \\
\hline & 7 & 0596 & 1 & & $3^{\circ}$ & 11 & Ene & 2003 & $6.04 / 28$ & & $\mathrm{X}$ & & 100 & \\
\hline & 8 & 0607 & & 1 & $3^{\circ}$ & 28 & Abr & 2003 & $6.01 / 11$ & & $\mathrm{x}$ & & 118 & \\
\hline \multirow{5}{*}{ UAPNEE 4} & 9 & 0679 & & 1 & $1^{\circ}$ & 14 & Ago & 2005 & $3.09 / 11$ & & $\mathrm{X}$ & & 140 & \\
\hline & 10 & 0696 & 1 & & $2^{\circ}$ & 27 & Ene & 2004 & $5.03 / 13$ & & $\mathrm{X}$ & & 127 & \\
\hline & 11 & 0703 & 1 & & $2^{\circ}$ & 15 & Abr & 2004 & $5.00 / 27$ & $x$ & & $x$ & 126 & \\
\hline & 12 & 0704 & & 1 & $1^{\circ}$ & 27 & Mar & 2005 & $4.02 / 07$ & & $\mathrm{x}$ & & 111 & \\
\hline & 13 & 0706 & & 1 & $2^{\circ}$ & 5 & Ago & 2004 & $4.08 / 19$ & $x$ & & $x$ & 121 & \\
\hline \multirow{3}{*}{ UAPNEE 5} & 14 & 0736 & & 1 & $3^{\circ}$ & 19 & Ago & 2003 & $5.09 / 08$ & & $\mathrm{x}$ & & 127 & \\
\hline & 15 & 0746 & & 1 & $2^{\circ}$ & 15 & Ene & 2004 & $5.04 / 11$ & $x$ & & $x$ & 113 & \\
\hline & 16 & 0750 & 1 & & $2^{\circ}$ & 6 & Jun & 2005 & $4.11 / 19$ & & $\mathrm{x}$ & & 112 & \\
\hline \multirow{4}{*}{ UAPNEE 6} & 17 & 0838 & & 1 & $2^{\circ}$ & 6 & Abr & 2004 & $5.01 / 12$ & & $\mathrm{X}$ & & 124 & \\
\hline & 18 & 0849 & 1 & & $2^{\circ}$ & 21 & Ago & 2004 & $5.08 / 03$ & & $\mathrm{X}$ & & 119 & \\
\hline & 19 & 0857 & 1 & & $1^{\circ}$ & 6 & Ene & 2006 & $3.05 / 13$ & & $x$ & & $*$ & INCUANTIFICABLE \\
\hline & 20 & 0861 & 1 & & $1^{\circ}$ & 11 & Ene & 2005 & $4.04 / 03$ & & $\mathrm{x}$ & & 117 & \\
\hline UAPNEE 7 & 21 & 0902 & & 1 & $2^{\circ}$ & 25 & Jun & 2004 & 4.11 & & $\mathrm{X}$ & & 111 & \\
\hline \multirow{6}{*}{ UAPNEE 9} & 22 & 0986 & & 1 & $3^{\circ}$ & 27 & Oct & 2003 & $5.07 / 15$ & & $\mathrm{X}$ & & 98 & \\
\hline & 23 & 0996 & 1 & & $1^{\circ}$ & 8 & Ago & 2005 & $3.09 / 12$ & & $x$ & & * & INCUANTIFICABLE \\
\hline & 24 & 1001 & & 1 & $1^{\circ}$ & 30 & Jun & 2005 & $3.09 / 12$ & & $x$ & & 108 & \\
\hline & 25 & 1004 & & 1 & $2^{\circ}$ & 15 & Ago & 2004 & $4.08 / 27$ & & $\mathrm{X}$ & & 98 & \\
\hline & 26 & 1006 & & 1 & $2^{\circ}$ & 22 & May & 2004 & $4.11 / 21$ & & $\mathrm{X}$ & & 126 & \\
\hline & 27 & 1007 & & 1 & $3^{\circ}$ & 14 & Ago & 2003 & $5.08 / 13$ & $\mathrm{X}$ & & $\mathrm{X}$ & 131 & \\
\hline UAPNEE 10 & 28 & 1047 & 1 & & $3^{\circ}$ & 5 & May & 2003 & $6.00 / 08$ & $\mathrm{X}$ & & $\mathrm{x}$ & 133 & \\
\hline \multirow{2}{*}{ UAPNEE 2 C } & 29 & 1084 & 1 & & $2^{\circ}$ & 22 & Dic & 2004 & $4.05 / 03$ & $x$ & & $x$ & 114 & \\
\hline & 30 & 1090 & & 1 & $2^{\circ}$ & 7 & Nov & 2004 & $4.05 / 20$ & & $x$ & & 116 & \\
\hline \multirow{2}{*}{ UAPNEE 2} & 31 & 1117 & 1 & & $2^{\circ}$ & 28 & Oct & 2004 & $4.06 / 28$ & & $x$ & & 130 & \\
\hline & 32 & 1136 & 1 & & $2^{\circ}$ & 27 & Feb & 2004 & $5.03 / 07$ & & $x$ & & 97 & \\
\hline \multirow{7}{*}{ JAPNEE 22} & 33 & 1167 & & 1 & $2^{\circ}$ & 17 & Mar & 2004 & $5.02 / 01$ & & $x$ & & 106 & \\
\hline & 34 & 1177 & 1 & & $2^{\circ}$ & 11 & Abr & 2004 & $5.01 / 16$ & & $x$ & & 99 & \\
\hline & 35 & 1181 & 1 & & $2^{\circ}$ & 17 & Jun & 2004 & $4.11 / 09$ & & $x$ & & 119 & \\
\hline & 36 & 1182 & & 1 & $2^{\circ}$ & 14 & Jul & 2004 & $4.10 / 11$ & & $x$ & & 94 & \\
\hline & 37 & 1200 & 1 & & $1^{\circ}$ & 17 & Ene & 2005 & $4.04 / 10$ & $x$ & & $x$ & 144 & \\
\hline & 38 & 1205 & 1 & & $2^{\circ}$ & 5 & Ago & 2004 & $4.09 / 13$ & $x$ & & $\mathrm{X}$ & 93 & \\
\hline & 39 & 1206 & 1 & & $2^{\circ}$ & 30 & Mar & 2004 & $5.01 / 27$ & $x$ & & $\mathrm{X}$ & 114 & \\
\hline \multirow[t]{3}{*}{ UAPNEE } & 40 & 1207 & 1 & & $2^{\circ}$ & 25 & Mar & 2004 & 5.0 & $x$ & & $x$ & 124 & \\
\hline & & & 18 & 22 & & & & & & & 29 & 11 & & \\
\hline & & & & 40 & & & & & & & & 40 & & \\
\hline
\end{tabular}

Nota: El número de folio permitió la identificación de la Tabla correspondiente al alumno valorado 
REVISTA DE INVESTIGACIÓN EDUCATIVA DE LA REDIECH N. 3

GRÁFICA 1. Escuela de preescolar en donde se implementó el Proyecto de Identificación Temprana

\section{Preescolares donde se implementó el Proyecto de Identificación Temprana}

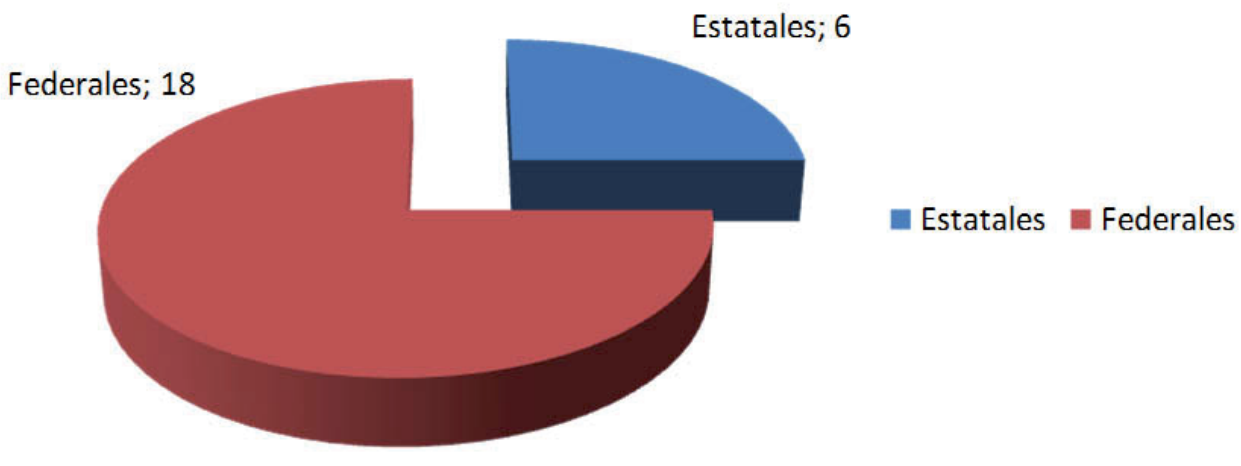

De un universo de 24 Preescolares, el $75 \%$ pertenecen al subsistema federal y el $25 \%$ al subsistema estatal

GRÁFICA 2. Alumnas y alumnos evaluados por medio de la Tabla de Observación y Desarrollo

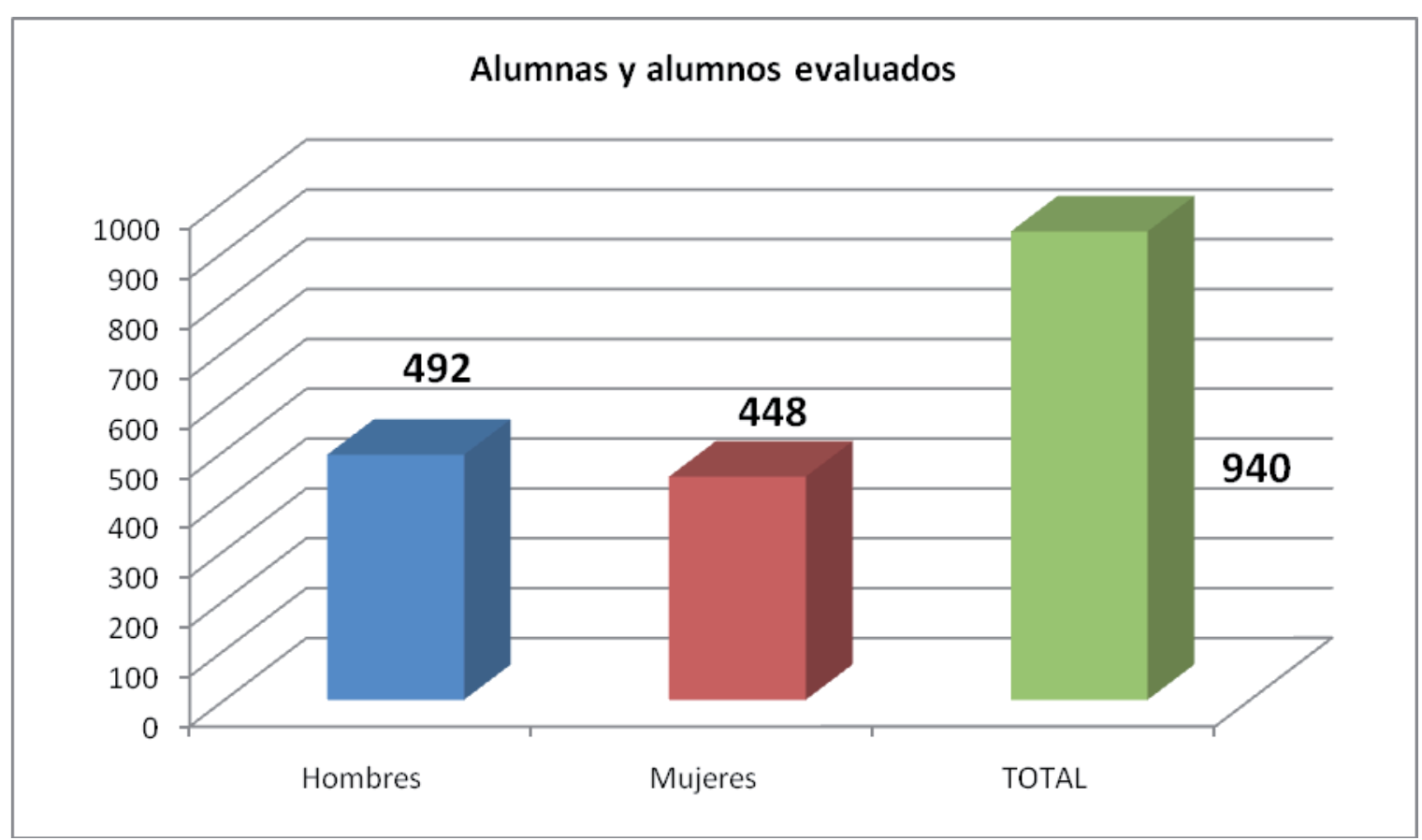

De un universo de 940 alumnas y alumnos que fueron valorados con la Tabla de Observación y Desarrollo, el 52\% fueron hombres y el $48 \%$ fueron mujeres. 
GRÁFICA 3. Alumnas y alumnos preseleccionados

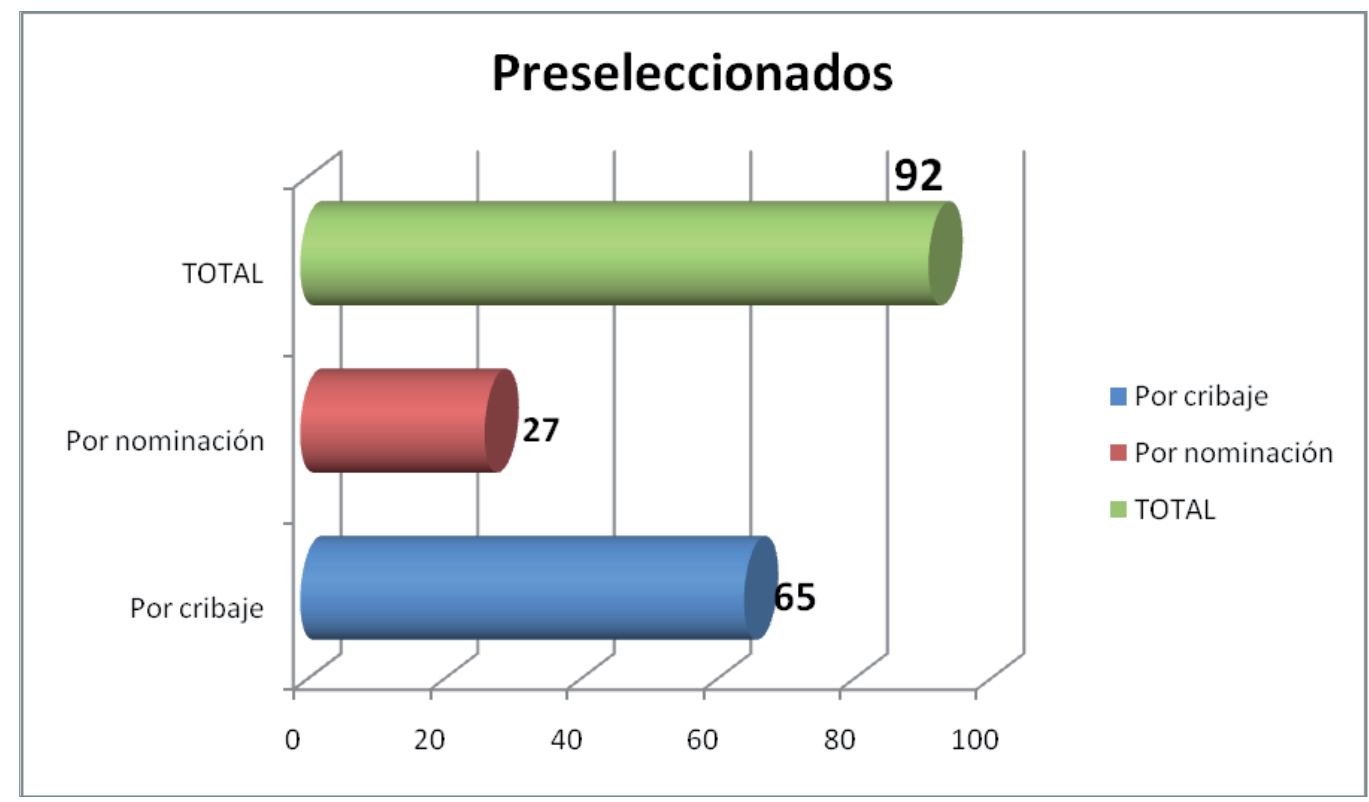

De los 940 alumnos que fueron valorados, únicamente el $9.78 \%$ fue preidentificado. De ésta población de 92 niños, un 70 \% son alumnos que pasaron la prueba de Observación y Desarrollo, el 30\% restante son alumnos que fueron nominados por criterio de las educadoras.

GRÁFICA 4. Instrumentos aplicados para corroborar el Coeficiente Intelectual de las y los niños preseleccionados

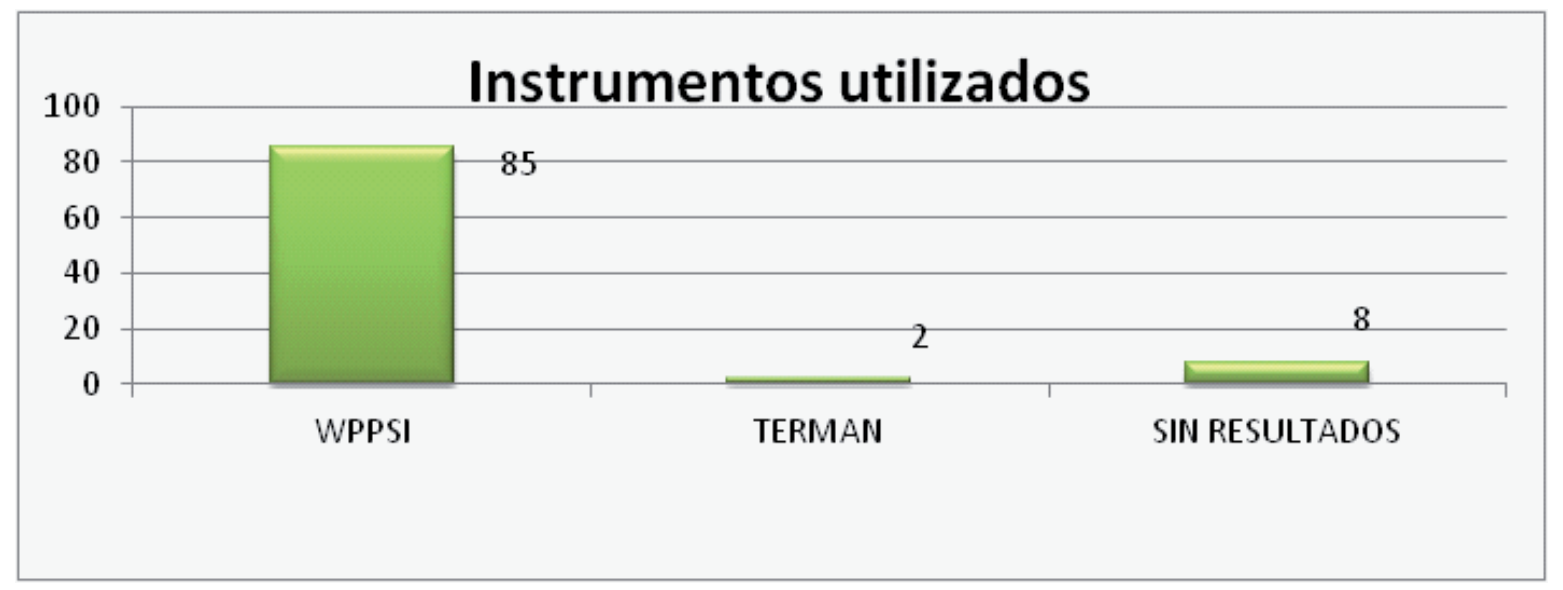

El 92\% de los alumnos preidentificados fue valorado con la Escala de Inteligencia para el nivel de preescolar. Un 2\% fue valorado por medio del Terman Merrill ya que por edad, no accedían al WPSSI. Existe un porcentaje de alumnos correspondiente al $8.6 \%$ que por diferentes razones no fue valorado (por baja, un alumno con Trastorno Generalizado del Desarrollo que no accedió, dos alumnos que no aceptaron ser evaluados y dos alumnos que no accedieron por edad. etc.) 
REVISTA DE INVESTIGACIÓN EDUCATIVA DE LA REDIECH N. 3

GRÁFICA 5. Resultados obtenidos en la valoración del Coeficiente intelectual

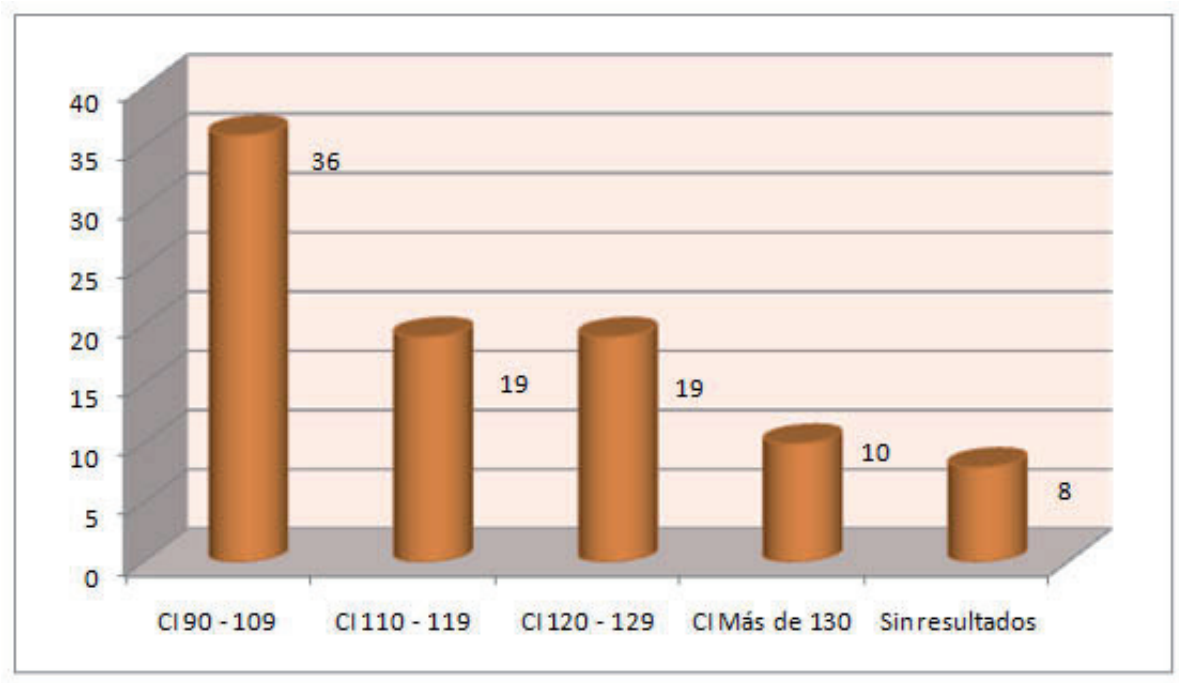

De un universo de 92 alumnos que fueron preseleccionados y valorados, el $39.1 \%$ se ubica con un CI entre 90-109, el $20.6 \%$ se ubica en el rango de 110-119, un $20.6 \%$ obtiene un coeficiente intelectual de $120-129$ y un $8.6 \%$ no arroja resultados.

* El criterio de superdotación con un CI equivalente a 130 o más, corresponde al $1.06 \%$ en comparación con la muestra total obtenida de 940 alumnos.

*El criterio de superdotación con un CI equivalente a 130 o más, corresponde al $10.8 \%$ en comparación con la muestra de 92 alumnos evaluados.

\section{Conclusiones}

Una vez que se concluyó el proceso de preselección y valoración de las y los alumnos con aptitudes sobresalientes en preescolar, se pudieron emitir las siguientes conclusiones.

De acuerdo con los resultados obtenidos, únicamente el $1.06 \%$ de la población de las y los alumnos tienen una superdotación intelectual, un porcentaje alejado de los resultados esperados del $2.2 \%$ propuesto por la bibliografía especializada en prevalencia de superdotación.

Los resultados de la Tabla de Observación y Desarrollo, permitió la identificación de alumnas y alumnos con un alto rendimiento, esto se comprobó al obtener un 52.17\% de niños y niñas que obtuvieron un CI superior a 110 puntos.

Como parte de las políticas nacionales y estatales, en la entidad se brindará atención educativa a todos aquellos alumnos y alumnas que hayan obtenido un CI igual o superior a 116 (39 casos) lo cual permitirá ampliar la cobertura en el nivel de preescolar. Esta población representa el $42.3 \%$ del total de alumnos que fueron preidentificados y el $4.1 \%$ del total de alumnos valorados con la Tabla de Observación y Desarrollo. 
En tres de los preescolares participantes que comparten características de deprivación social, el instrumento denominado Tabla de Observación y Desarrollo, arrojó resultados no satisfactorios, al no encontrar ningún alumno que pasara el cribaje. La posible explicación es que el nivel socioeconómico y cultural y las condiciones contextuales en las que viven las familias, son adversas para contestar los datos específicos que requiere el llenado de la Tabla.

Existe un alto número de alumnas y alumnos que fueron nominados por criterio de las educadoras y que no habían pasado el cribaje. Los resultados arrojan datos que permiten corroborar que la nominación directa cobra validez en el proceso de preselección en preescolar, ya que un alto número de estos alumnos obtuvieron un CI superior a los 130 (6 de los 10 alumnos identificados).

Con respecto a las diferencias de género, se puede concluir que en la experiencia de la entidad, el 50\% de la población identificada como superdotada, corresponde al sexo masculino y el otro $50 \%$ al sexo femenino.

La experiencia de trabajo que se vivió con el Proyecto de Identificación Temprana de niñas y niños sobresalientes en Preescolar en el ciclo 2008 - 2009, fue el primer acercamiento de los servicios de educación especial en este nivel -USAER y UAPNEE-, y marca como retos: 1) iniciar la generalización en otros servicios y preescolares, 2) continuar con las acciones de evaluación psicopedagógicas de las y los alumnos identificados, 3) diseñar las estrategias de intervención educativa.

\section{Situación actual}

A un año y medio de distancia del haber concluido el proyecto de investigación, se reportan como beneficios el haber continuado con las acciones de generalización en los diferentes servicios de educación preescolar que atienen niñas y niños con aptitudes sobresalientes. Durante el ciclo escolar siguiente a la investigación se dio paso a la generalización ampliando la cobertura en las regiones de $\mathrm{Cd}$. Juárez, Parral, Delicias y Cuauhtémoc.

Actualmente Chihuahua reporta la atención educativa de 227 niñas y niños con aptitudes sobresalientes en preescolar, cuando inicialmente fueron detectados durante la investigación 39 casos. Esto habla de un incremento en cobertura en el nivel y los servicios de educación especial.

A nivel nacional no se ha generado una propuesta que dé respuesta educativa en la identificación e intervención para niñas y niños sobresalientes en preescolar, lo cual permite colocar al estado de Chihuahua, como pionero en la atención de esta población, gracias al trabajo elaborado en el Proyecto de Investigación.

\section{Notas}

1 Centro de identificación, seguimiento, formación e investigación en el campo de la superdotación y el talento. Valladolid, España.

\section{Referencias}

Benito, Yolanda (2009). Conceptualización: sobredotación intelectual $y$ talentos. Documento digitalizado.

SEP (2006) Propuesta de Intervención: Atención educativa a niños y niñas con aptitudes sobresalientes. México; Secretaría de Educación Pública.

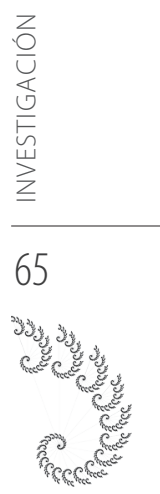


